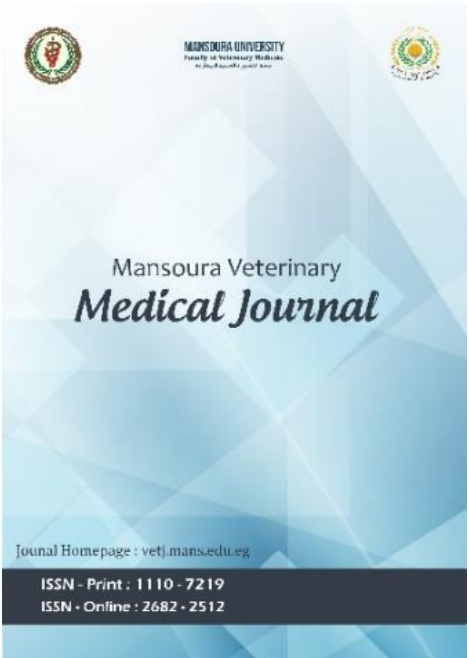

\title{
The efficacy of clove and thyme against experimentally induced Candidiasis in broilers
}

Ghada Abdel fatah, Aza Hassan, Rasha Saleh, Magdy Amer

To cite this article: Ghada Abdel fatah, Aza Hassan, Rasha Saleh, Magdy Amer.The efficacy of clove and thyme against experimentally induced Candidiasis in broilers. Mansoura Veterinary Medical Journal 2020; 21, 2: 2531.

To link to this article: https://doi.org/10.35943/mvmj.2020.21.2.0205

Published online: 25 June 2020

Submit your article to this journal 


\title{
The efficacy of clove and thyme against experimentally induced Candidiasis in broilers
}

\author{
Ghada Abdel fatah ${ }^{1 *}$, Aza Hassan ${ }^{1}$, Rasha Saleh², Magdy Amer ${ }^{3}$ \\ ${ }^{1}$ Animal Health Research Institute, Mansoura, Egypt \\ ${ }^{2}$ Department of Physiology, Faculty of Veterinary Medicine, Mansoura University, Mansoura 35516, Egypt \\ ${ }^{3}$ Department of Pharmacology, Faculty of Veterinary Medicine, Mansoura University, Mansoura35516, Egypt
}

\section{ARTICLE HISTORY}

Received: 21.12.2019

Revised: 17.05 .2020

Accepted: 18.05 .2020

Address correspondence to Ghada Abdel fatah; Tel: +2001092953888; E-mail: agdyj5f50@yahoo.com

\section{ABSTRACT}

\begin{abstract}
Objectives: The present study was designed to investigate the efficacy of clove powder and thyme oil against experimentally-induced candidiasis in broiler chicken.

Design: Randomized controlled experimental study.

Animals: One hundred and twenty five chicken of one month old were used in this study Procedures: Chicken were randomly allocated into 5 equal groups of 25 bird each. The first group was used as a negative control which received no treatment. The second group was experimentally infected with candida (C. albicans), and served as a positive control. Meanwhile, the third, fourth and fifth groups were also experimentally infected with candida, in addition to receiving clove powder (2\%), thyme oil $(200 \mathrm{ml} / \mathrm{kg})$ in diet and fluconazole $(5 \mathrm{mg} / \mathrm{kg})$ respectively, for 10 days after the appearance of the symptoms of candida. The symptoms included delayed gastrointestinal transit times, static crop or sour crop, anorexia and regurgitation. The normal thin wall of the crop become thickened with a complete crop stasis.

Results: Administration of clove powder and thyme oil improved the symptoms induced by $C$. albicans and increased PCV \%, ALT and AST level, with a decrease in erythrocyte count and total leukocyte count. Clove powder increased creatinine levels, while thyme oil decreased them. The tested plants induced marked histopathological alteration in crop, lung, intestine and gizzard of treated birds.
\end{abstract}

Conclusion and clinical relevance: Clove powder and thyme oil can be used as an alternative medicine for the treatment of candidiasis in chicken.

Keywords: : Clove powder, Thyme oil, Candida, Chicken

\section{INTRODUCTION}

Poultry is considered as one of the most important source of animal protein of high biological value all over the world. The meat of poultry provides protein of high biological value, minerals and vitamins [1]. The contamination of poultry diet with mycotoxins-producing fungi is considered as one of the serious problems confronting poultry breeders worldwide [2].

Mycotic infections are common in poultry farms. A common pathogen is candida albicans, which is considered a part of the normal flora of mucous membranes. It is present in the environment and can invade animals and humans [3]. Chickens and other avian species can be affected by Candida albicans causing Candidiasis. This fungus induces its lesions mainly in the crop of the affected bird (e.g. crop mycosis or thrush). Candidiasis affects mainly the young bird (chicks) resulting in stunning and death. Candidiasis is classified according to the degree of severity into superficial (mucosal) or systemic candidiasis [4].

The control of mycotic infections necessitates the use of antifungal drugs. The introduction of new antimycotic agents is always required due to the increase of mycotic resistance to the usual antifungal drugs. The search for new natural antimycotic agents is important to overcome the treatment cost, the fungal resistance to the synthetic antifungal agents and the narrow spectrum and fungi static effect of the available antimycotic agents [5]. Plants are one of the most important and traditional sources of medicines. Several drugs used in modern system are of natural source (natural herbs). The use of herbal agents has many advantages as they are safe, economic, effective and easy to administrate [6].

Thymus vulgaris is a medicinal herb that belongs to the family Lamiaceae. Thymus has many useful effects as antiseptic, antispasmodic, antimicrobial and expectorant [7]. The antimicrobial activity of thymus is attributed to its active 
component (thymol and carvacrol). In addition, the active components improve the viability and growth of treated birds due to its antibacterial, antimycotic and antioxidant activities. Clove is a plant that is widely cultivated in many countries, though earlier production of the plant was in China. Its antimicrobial potential was established when its essential oil extracts killed many gram-positive and gram-negative organisms including some fungi. The antimicrobial activity of clove is attributable to eugenol, oleic acids and essential oils. Clove and its essential oil is one of the plant extract that could improve growth performance in broilers [8].

The present study was therefore carried out to investigate the efficacy of thyme essential oil and clove powder against experimentally induced candidiasis in broiler chicken.

\section{MATERIALS AND METHODS}

\subsection{Animals}

One hundred and twenty five (125) clinically healthy nonvaccinated chicks with an average body weight of $45 \pm 3 \mathrm{gm}$ were purchased from a local poultry farm in Gamasa city, Dakahlia Governorate and were reared for one month before the start of the experiment. Two weeks prior to the start of the experiment, the chickens were accommodated at the poultry laboratory at Animal Health Research Institute, Mansoura. The chickens were kept under a good hygienic condition and were fed on a ration containing (Yellow corn, choline chloride $60 \%$ (2240), soybeans $48 \%$, limestone powder, mix vitamins (2955), table salt, corn gluten $60 \%$, calcium phosphate two, soy vegetable oil and sunflower, mixture of mineral salts (2459) and D L Methionine). The chickens were divided into five groups of 25 birds each.

\subsection{Induction of candidiasis}

Induction of $C$. albicans was achieved using a $0.1 \mathrm{ml}$ suspension containing $3 \times 10^{8} \mathrm{C}$. albicans viable cells [9]. For all chickens, a cotton swab containing the suspension was rolled twice inside the mouth cavity of the birds and was repeated twice daily with $48 \mathrm{hrs}$ intervals. The infected chickens were examined carefully for the presence of the lesions in the oral cavities before starting of treatment protocol.

\subsection{Experimental design}

After 72 hours of the start of the experiment, chickens were randomly allocated into five equal sized groups (each of 25 birds). The first group served as non-infected group and received no treatment (a negative control). The second group was infected non-treated group (a positive control), as birds in this group was experimentally infected with Candida and received no antifungal agents. The third group was infected with candida and received a basal diet mixed with thymol oil (Sigma Aldrich, Germany) at a rate of $200 \mathrm{ml} / \mathrm{Kg}$ diet for two weeks, according to the method used previously [10]. The fourth group was infected with candida and received a basal diet amended with $2 \%$ clove powder ( $20 \mathrm{gm} / \mathrm{kg}$ diet) for two weeks, according to a previously applied protocol [11]. On the other side, chicken of the fifth group were infected with candida and received fluconazole (SEDICO Company, 6 October City, Egypt) orally in drinking water at a dose of $5 \mathrm{mg} /$ kg BW once daily for 10 days [12].

\subsection{Blood sampling}

Blood samples were collected from five chickens of each group after euthanasia after the first week (T1), second week (T2) and third week (T3) of antifungal administration. Samples were collected into two test tubes. The first tube was mixed with ethylene diamine tetra acetic ( $2 \%$ EDTA solution, 1-2 drops per $\mathrm{ml}$ of whole blood) and was used for hematological tests according to Tehraniet al. [13], while the second blood sample was collected without the use of anticoagulant in non-heparinized test tubes and was centrifuged at 3000/ $15 \mathrm{rpm}$ to get a clear serum samples.

\subsection{Hematological parameters}

Total erythrocyte count (million/ $\mathrm{mm}^{3}$ ) was determined according to the method described by Haile and Chanie [14]. Hemoglobin was determined colorimetrically according to the method described by Tehrani et al. [13]. Total leucocyte count (thousand $/ \mathrm{mm}^{3}$ ) was determined according to the method described by Frankič et al. [15].

\subsection{Biochemical analysis}

\subsubsection{Determination of serum transaminases}

The concentrations of alanine aminotransferase and aspartate amino transferase in serum were measured colorimetrically according to the method described by Frankičet al. [15].

\subsubsection{Determination of creatinine and uric acid}

Levels of serum creatinine and serum uric acid were determined by a colorimetric method as previously described [15].

\subsection{Histopathological examination}

The crop, lung, intestine and gizzard samples were collected from all group at the third week of drug administration. Samples were examined histopathologically according to the method described by Banchrof et al. [16]

\subsection{Statistical analysis}

SPSS, version 17, (Inc., Chicago, IL. USA) computer software was used for statistical analysis of the data. Data were expressed as means \pm standard error. The effects of using antifungal agents on each variable measured were analyzed using general linear model GLM-repeated measures. When there was a significant effect, one-way ANOVA with post hoc Duncan multiple comparison tests were used to detect the specific variations and differences between each pair of the groups, and the level of significance was set at $\mathrm{P}<0.05$ [17]. 


\section{RESULTS}

\subsection{Effect of the clove, thyme and fluconazole on hemogram}

\subsubsection{Total erythrocyte count}

The effect of oral administration of clove powder, thyme oil and fluconazole on the total erythrocyte count is presented in figure 1 . The erythrocyte counts of treated groups (all groups except the first) showed no significant alterations at $\mathrm{T} 1$ (one week post administration of the antifungal agents) from the control group (Figure 1). At T2 (two weeks post administration of the antifungal agents), erythrocyte count in treated groups decreased significantly compared to the negative control group. The infected nontreated group (second) was the most affected one and had the lowest erythrocyte count. At T3 the erythrocyte counts were significantly lower in thyme-treated compared to that of the positive control (second) group (Figure 1a).

\subsubsection{Hemoglobin}

The effect of the oral administration of clove powder, thyme oil and fluconazole once daily for three weeks on $\mathrm{Hb}$ levels of chickens is presented in figure $1 \mathrm{~b}$. The data showed no significant variations in levels of $\mathrm{Hb}$ between groups or within the same group at different time points.

\subsection{3. $P C V \%$}

The effect of oral administration of clove powder, thyme oil and fluconazole once daily for three weeks on PCV \% of chickens is presented in figure 3. At T1 thyme-treated group recorded a higher \% of PCV compared to that of the negative and positive control group, and clove-treated group. At T2, the negative control group had the lowest \% of PCV compared to the other groups except fluconazole-treated group. Moreover, \% of PCV of fluconazole-treated group was lower than those of both positive control and clove-treated group. At T3, there were no significant variations in PCV \% between the different groups (Figure 1c).

\subsection{4. ffect of clove, thyme and fluconazole on total leucocyte count}

The effect of the oral administration of clove powder, thyme oil and fluconazole once daily for three weeks on total leucocyte count of chickens is presented in figure1d. At T1 the leucocyte counts did not significant changes between different groups when compared to the control group. At T2, the total leucocyte counts decreased in clove, thyme and fluconazole-treated groups when compared to both control groups. The difference between both clove and thymetreated groups on one side and fluconazole-treated group on the other side was significant. At T3, there was a significant decrease in total leucocyte count in clove, thyme and fluconazole-treated groups, compared to the two control groups.

\subsection{Effect of clove, thyme and fluconazole on liver function}
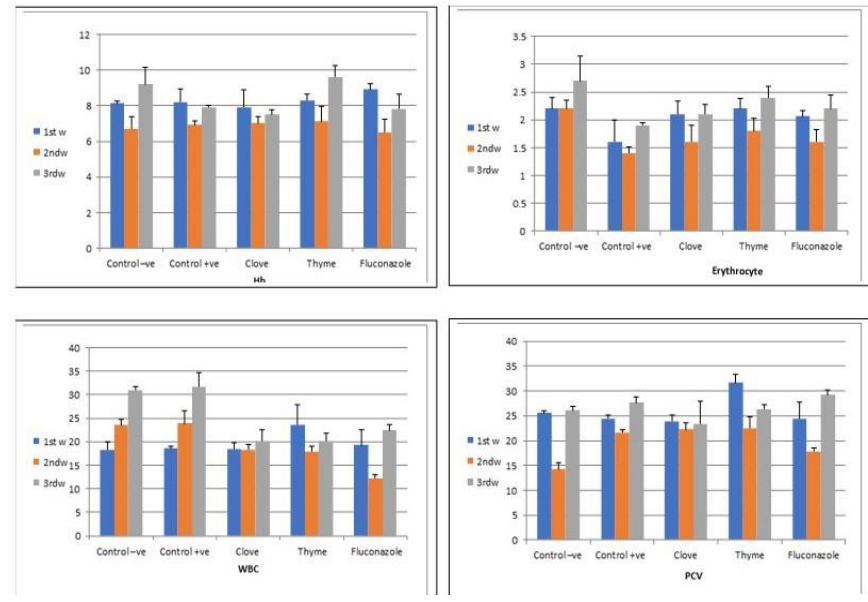

Figure 1. Effect of oral administration of clove powder, thyme oil and fluconazole on RBC, $\mathrm{Hb}, \mathrm{PCV} \%$ and WBCs of chicks infected with candida at different time intervals.

\subsubsection{Serum Alanine Amino transferase (ALT)}

The effect of the oral administration of clove powder, thyme oil and fluconazole once daily for three weeks on serum levels of ALT in chicken is presented in figure 5. At alltime points, both the positive control group and fluconazoletreated group showed higher values of serum ALT compared to the other three groups (Figure 2).

\subsubsection{Serum Aspartate Aminotransferase (AST)}

The effect of the oral administration of clove powder, thyme oil and fluconazole once daily for three weeks on serum levels of ALT in chicken is presented in figure 6. At T1, the administration of both clove powder and thyme oil decreased the serum levels of AST compared to both positive control group and fluconazole-treated group, however the difference was significant when compared to the positive control group only (Figure 6). At T2 and T3, clove and thyme oil-treated groups displayed significantly lower levels of serum AST when compared to both positive control and fluconazole-treated groups (Figure 2).

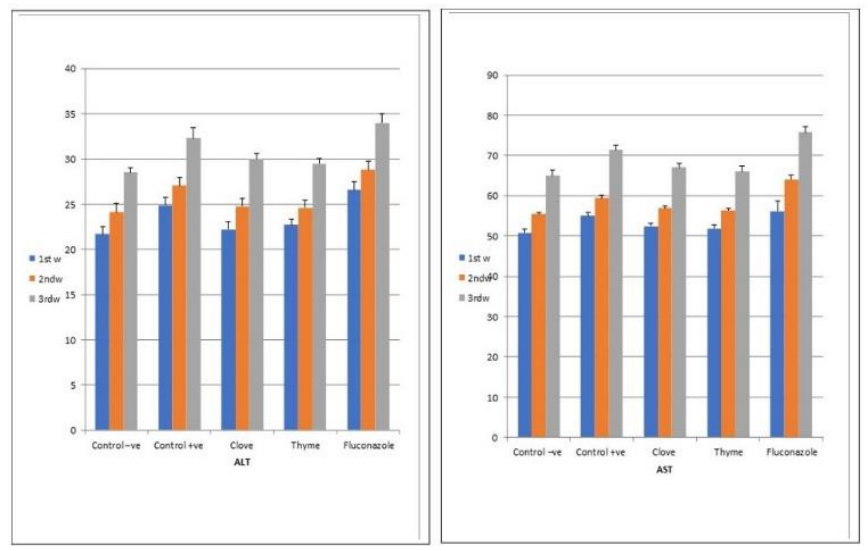

Figure 2. Effect of oral administration of clove powder, thyme oil and fluconazole on ALT and AST of chicks infected with candida at different time intervals. 


\subsection{Effect of clove, thyme and fluconazole on kidney function}

\subsubsection{Serum uric acid}

The effect of oral administration of clove powder, thyme oil and fluconazole once daily for three weeks on levels of serum uric acid in chicken is presented in figure 7. No significant variations were recorded in serum uric acid values between clove and thyme-treated group at different time points of sampling, whereas fluconazole-treated group displayed significantly higher values of serum uric acid levels at T1, T2 and T3 compared to either positive control group or clove and thyme-treated groups (Figure 3 ).

\subsubsection{Serum creatinine}

The effect of oral administration of clove powder, thyme oil and fluconazole once daily for three weeks on serum creatinine levels in chicken is presented in figure 8 . At all-time points of the experiment both positive control group and fluconazole-treated groups showed higher values for serum levels of creatinine compared to both clove and thymetreated groups of chicken (Figure 8). It was also noticed that the administration of thyme oil induced a further significant reduction in serum levels of creatinine compared to clovetreated groups (Figure 3).

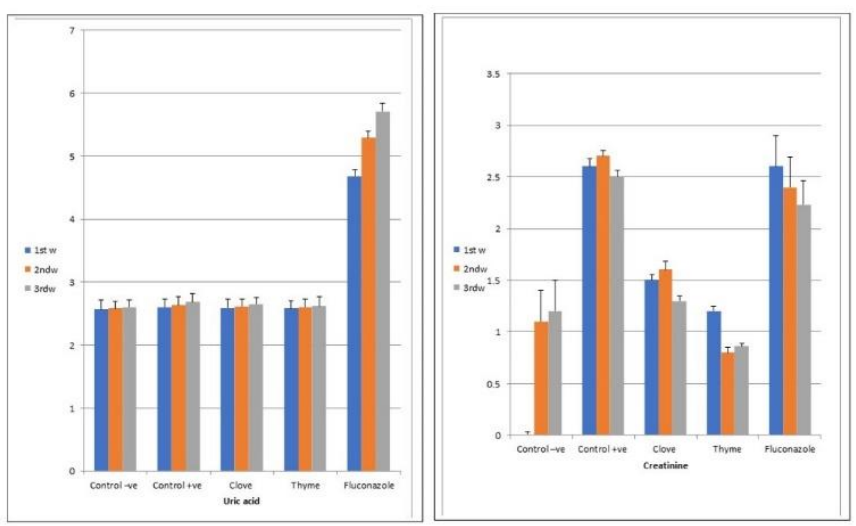

Figure 3. Effect of oral administration of clove powder, thyme oil and fluconazole on uric acid and creatinine of chicks infected with candida at different time intervals.

\subsection{Histopathology}

Results reflected that microscopic pictures of crop sections showed normal histological picture in negative control group, hyperkeratotic lesion, massive heterophils and eosinophils infiltration in mucosa in positive control group. Meanwhile, mild heterophils and eosinophil infiltration was detected in mucosa from fluconazole and thyme-treated groups. Microscopic pictures of crop sections showed presence of yeast and pseudohyphae in hyperkeratotic lesion in the positive control group only. These results are presented in figure 4 and 5.

The initial lesions comprised necrotic plaques followed by thickening and corrugation of crop mucosa. The thickening and corrugation became well marked after 10 days of infection and continued till the end of the experimental period. Histologically, hyperkeratosis and parakeratosis were the salient changes up to T2 (14 ${ }^{\text {th }}$ day) but thereafter thickening of crop mucosa was the result of parakeratosis, subepithelial congestion and edema.

According to figure 6-8, there were marked improvement in crop of group treated with thyme and fluconazole compared to other groups. Also, lung of chicken treated with thyme and fluconazole retained normal histopathological pictures. Gizzard showed improvement in thyme and fluconazole-treated group. The enteric wall retained normal histological appearance in fluconazole, thyme and clove-treated groups.
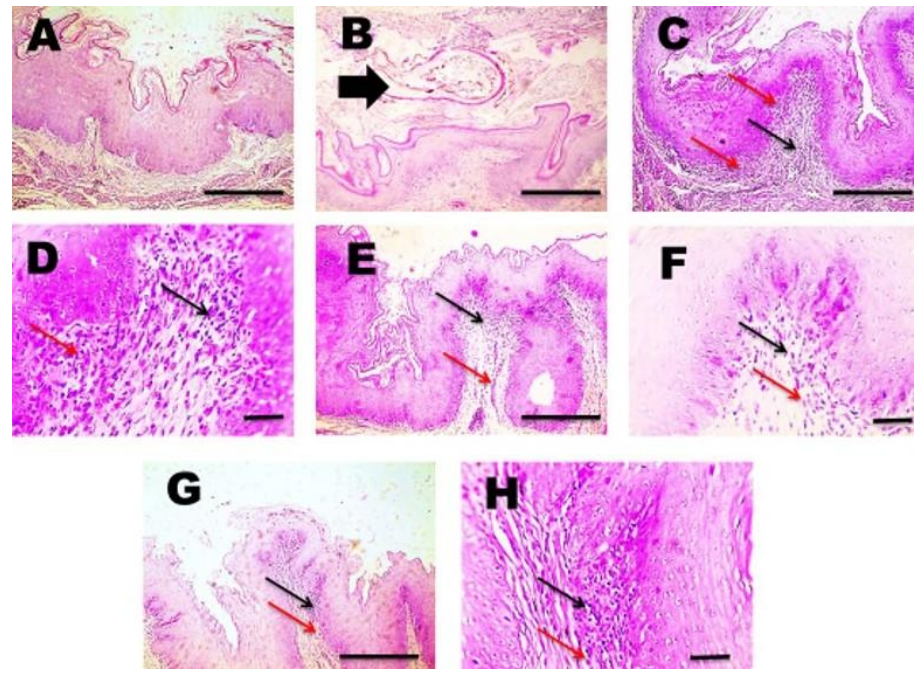

Figure 4. Microscopic pictures of crop sections showing normal histological picture in negative control group (A), hyperkeratotic lesion (thick arrow) (B), massive heterophils (thin black arrow) and eosinophils (thin red arrow) infiltration in mucosa $(C \alpha D)$ in positive control group. Meanwhile, mild heterophils and eosinophil infiltration is detected in mucosa (thin black arrow) from fluconazole $(\mathrm{E} \alpha \mathrm{F})$ and thyme $(\mathrm{G} \alpha \mathrm{H})$ treated groups. $\mathrm{H} \alpha \mathrm{E}, \mathrm{X:100}$ bar100. (A, B, C, E, G) X: 400 bar 50 (D, F, H).
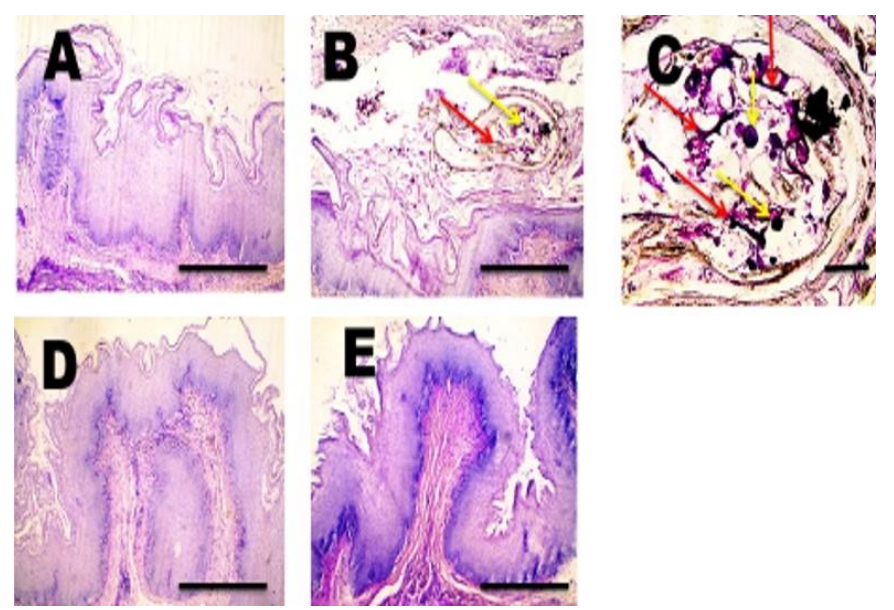

Figure 5. Microscopic pictures of crop sections showing presence of yeast (thin black arrow) and pseudohyphae (thin red arrow) in hyperkeratotic lesion $(\mathrm{BaC})$ in the positive control group only. PAS, X:100 bar 100 (A, B, D, E) X: 400 bar 50 (C). 

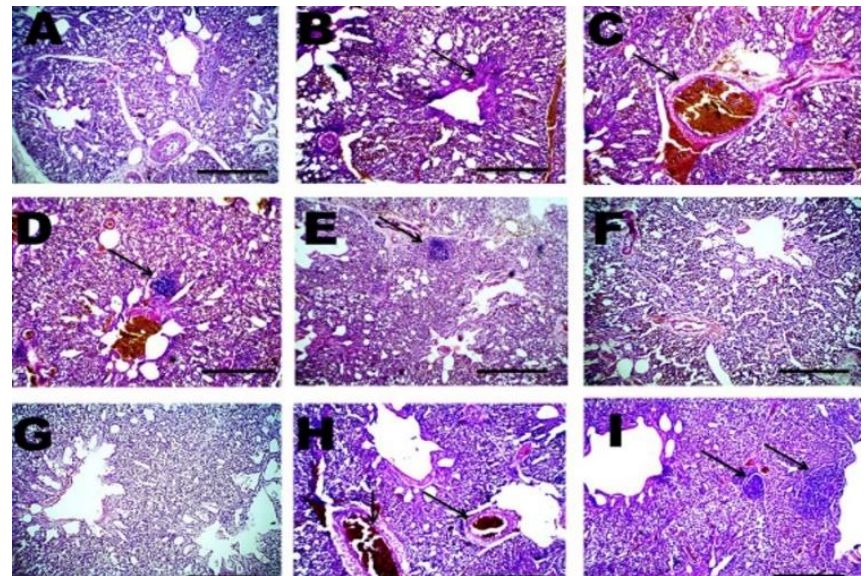

Figure 6. Microscopic pictures of lung sections showing normal histology of parabronchi, blood vessels and interstitial tissue in the negative control group (A), narrowing in lumen of parabronchus due to fibrosis in wall of parabronchus (black arrow) (B), congested blood vessel (black arrow) (C), small multifocal lymphocytic cells aggregation in pulmonary tissue (black arrow) (D $\alpha E)$ in the positive control group, pulmonary tissue retained normal histological pictures in fluconazole $(\mathrm{F})$ and thyme-treated groups $(\mathrm{G})$, congested blood vessels (black arrows) $(\mathrm{H})$ with small multifocal lymphocytic cells aggregation in pulmonary tissue (black arrows) (I) in clovetreated group. $\mathrm{H \alpha E}$ X: 100 bar 100.
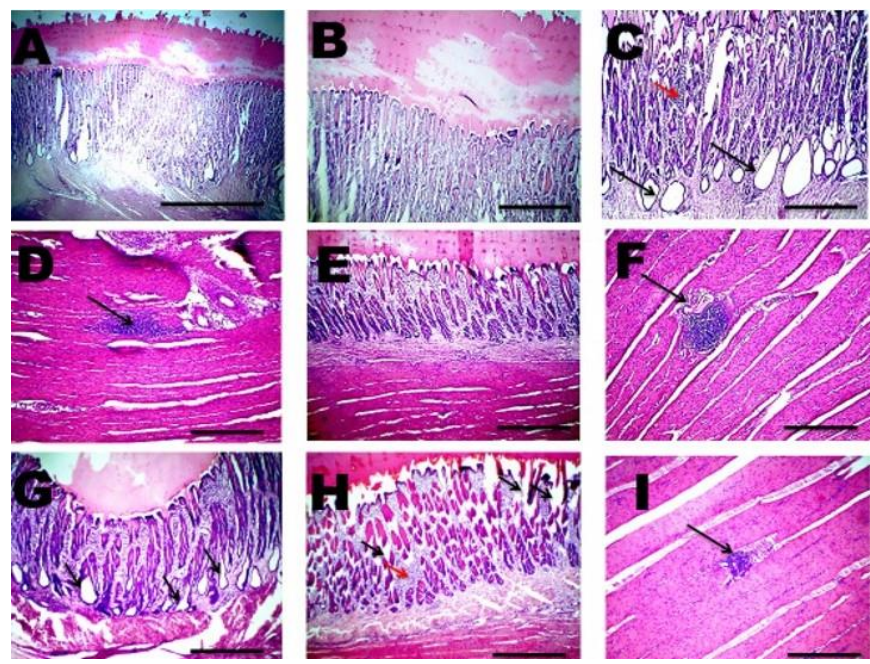

Figure 7. Gizzard sections show normal histology in the negative control group $(\mathrm{A} \alpha \mathrm{B})$, small focal lymphocytic aggregation (red arrow) and cystic dilation of glands in gastric mucosa (black arrow) (C) with small multifocal lymphocytic cells aggregation in muscular layer (black arrow) (D) in the positive control group, normal gastric mucosa $(E)$, with small focal lymphocytic cells aggregation in muscular layer (black arrow) (F) in fluconazole-treated group, cystic dilation of glands in gastric mucosa (black arrow) in thyme-treated group (G), small focal lymphocytic aggregation (red arrow) and mild edema in gastric mucosa (black arrows) $(\mathrm{H})$ with small focal lymphocytic cells aggregation in muscular layer (black arrow) (I) in clove-treated group. $\mathrm{H} \alpha \mathrm{E}, \mathrm{X}: 40$ (A) bar 200 and X:100 (B-I) bar 100.
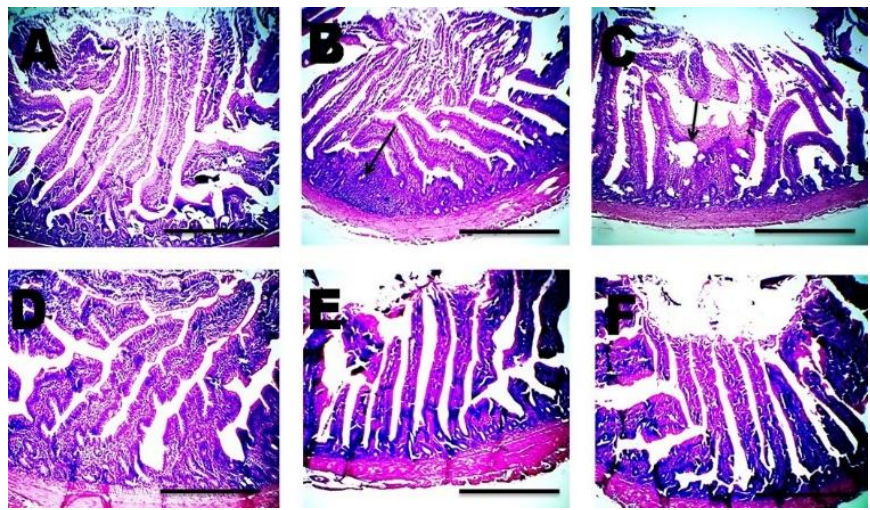

Figure 8. Normal histology of intestinal villi and lamina propria in the negative control group (A); focal lymphocytic aggregation (black arrow) (B); with fusion of villi (black arrow) (C) in the positive control group. The enteric wall retained normal histological appearance in fluconazole (D), thyme (E) and clove (F) treated groups. $\mathrm{H} \alpha \mathrm{E}, \mathrm{X}: 40$ bar 200.

\section{DISCUSSION}

Fungal infections have been increased recently due to a growing number of pathogenic fungi that have serious side effects [18]. Nevertheless, few anti-fungal medicines are still available for the treatment of these infections. Additionally, there has been a growing interest to seek alternatives to chemical compounds. Hence, using natural therapeutic agents that have antifungal efficacy could be effective with potential side effects. In this context, thyme (Thymus vulgaris L.), is one of the most common medicinal plant that grows in the Mediterranean regions, that has received a particular attention due to its antibacterial and antioxidant activities against large number of pathogenic microbial organisms [19]. Clove buds, a popular class of kitchen spice referred to as 'the champion of spices', have been extensively studied and reported to possess different beneficial health and pharmacological effects, such as antidiabetic, hypolipidemic, gastro-protective and immunomodulatory activities [20]. In the present study, the use of clove powder and thyme oil improved the clinical symptoms induced by $C$. albicans compared to the infected non-treated group (positive control). This finding confirms the potential antifungal activity of clove powder and thyme oil as previously mentioned. Other research word found similar results that eugenol and clove oil could have antifungal effect against $C$. albicans [21, 22].

Blood parameters are a good benchmark for judging the health of an animal and are broadly viewed as crucial indicators of the nutritional and physiological status of birds and animals [23]. In this regard, the findings of the present study showed that the used of clove powder and thyme oil increased erythrocyte count and PCV in treated birds, and produced no changes in hemoglobin. These results agree with previously reported findings in that chicken treated with thyme and clove showed improved blood parameters such as $\mathrm{PCV} \%$ without alteration in $\mathrm{Hb} \%[11,1825]$. 
Hepatic function of birds can be evaluated by measuring serum concentration of liver enzymes (ALT, AST) since their synthesis occurs in the liver. The disruption of transaminases form normal values denotes hepatotoxicity because they are involved in detoxification process, biotransformation and biosynthesis of energetic macromolecules for different essential function. Increases in serum levels of AST have been reported in hepato-cellular diseases [26]. In the present study, serum ALT and AST increased in all treated groups compared with negative control. However, the increase in case of fluconazole-treated group was higher than that of both clove and thyme-treated groups. These findings are supported by the findings reported by Abdelaziz et al. [27] who found that adding thyme oil (Thymus vulgaris) at a dose of $250 \mathrm{mg} / \mathrm{Kg}$ diet could increase ALT activity. This means that using herbal antifungal agent increased liver activities without any adverse effect. This could indicate that both thyme oil and clove powder can be alternatives to fluconazole in treatment of candida albicans in chicken with similar antifungal activity and lower side effects. The finding of the present word also agree with those reported previously [28] and [29] in that fluconazole increased serum ALT level in treated group. These resulted illustrated also that, after use of fluconazole has stopped all the liver parameters returned to their normal levels.

Our result showed that there was no significant variation in uric acid in clove and thyme-treated groups, and that there was a mild increase in fluconazole-treated group. Moreover, there was a prominent increase in serum creatinine levels in fluconazole-treated group, and a mild increase in clovetreated group and a rather decrease in thyme-treated group. These results are supported by those of Saleh et al. [11] who found that dietary supplementation of thyme oil did produce significant variations in serum uric acid levels in broiler chicken. In addition Liu et al. [30] found that uric acid level increased significantly after 6 weeks of treatment with fluconazole in patients suffered from cryptococcal meningitis.

The results regarding the microscopic picture of crop in negative control group showed normal histopathological picture. On the other hand, the crop of positive control group revealed a marked change with the presence of yeast and pseudo hyphae. Addition of thyme oil and clove powder to the diets of infected chicken improved crop lesions and the histopathological picture markedly compared to the use of fluconazole.

It is likely that the occurrence of edema and congestion may be a response to the fungal toxins. This needs a further investigation. Fungal elements could be demonstrated in the hyperkeratotic epithelium up to the $14^{\text {th }}$ day. It has been reported in chickens and turkeys that fungal invasion was restricted to the superficial layers of epithelium and did not cross the basement membranes [31].

\section{Conclusion}

The findings of the current experiment demonstrate that clove powder and thyme oil can be used as alternative medication for treatment of Candidiasis in chicken with a marked efficacy and a low side effect.

\section{Conflict of interest statement}

The authors declare that there is no any conflict of interest in the current research work.

\section{Research ethics committee permission}

The current research work was conducted according to standards of Research Ethics Committee, Faculty of Veterinary Medicine, Mansoura University.

\section{Authors' contribution}

\section{All authors contributed equally}

\section{REFERENCES}

[1] Ovesen L, Brot C, Jakobsen J. Food contents and biological activity of 25hydroxyvitamin D: a vitamin D metabolite to be reckoned with?. Ann Nutr Metab 2003;47:107-13. https://doi.org/10.1159/000070031

[2] Manafi M, Murthy H, Swamy H. Evaluation of different mycotoxin binders on aflatoxicosis in broiler breeders induced with aflatoxin B: effects on biochemical and immunological parameters. Agric Environ Sci 2012;12:429-33.

[3] Quinn PJ, Markey BK, Leonard FC, Hartigan P, Fanning S, Fitzpatrick E. Veterinary microbiology and microbial disease: JWS; 2011.

[4] Jayatilake J. A review of the ultrastructural features of superficial candidiasis. Mycopathologia 2011;171:235-50. https://doi.org/10.1007/s11046-010-9373-7

[5] Rapp RP. Changing strategies for the management of invasive fungal infections. Pharmacotherapy: Pharmacotherapy 2004;24:4S-28S. https://doi.org/10.1592/phco.24.3.4S.33151

[6] Ramesh P, Okigbo R. Effects of plants and medicinal plant combinations as anti-infectives. Afr J.Pharm.Pharmacol 2008;2:130-5.

[7] Ciftci M, Guler T, Simsek UG, Ertas ON, Dalkilic B, Bicer Z. The effect of Thymus vulgaris L. oil as growth promoter in broilers. Gov Vet J 2009;86:930-2.

[8] Ordonez G, Llopis N, Penalver P. Efficacy of eugenol against a Salmonella enterica serovar enteritidis experimental infection in commercial layers in production. J Appl Poult Res 2008;17:376-82. https://doi.org/10.3382/japr.2007-00109

[9] Chami N, Chami F, Bennis S, Trouillas J, Remmal A. Antifungal treatment with carvacrol and eugenol of oral candidiasis in immunosuppressed rats. Braz J Infect Dis 2004;8:217-26. https://doi.org/10.1590/S141386702004000300005

[10] Hussein HZ. Activity of pomegranate peels and clove powders in detoxification of aflatoxin B1 and ochratoxin A from contaminated poultry diet. JPPM 2015;6:1. https://doi.org/10.4172/21577471.1000249

[11] Saleh N, Allam T, El-Latif A, Ghazy E. The effects of dietary supplementation of different levels of thyme (Thymus vulgaris) and ginger (Zingiber officinale) essential oils on performance, hematological, biochemical and immunological parameters of broiler chickens. Glob.Vet 2014;12:736-44.

[12] Rochette F, Engelen M, Vanden Bossche $H$. Antifungal agents of use in animal health-practical applications. J Vet Pharmacol Ther 2003;26:3153. https://doi.org/10.1046/j.1365-2885.2003.00457.x

[13] Tehrani A, Javanbakht J, Askari S, Hassan M, Solati A, Golami S, et al. Haematological studies on broiler chickens fed with different levels of Artemia Urmiana. J Biotechnol Biomater 2012;2:10.4172. https://doi.org/10.4172/2155-952X.1000138

[14] Haile $Y$, Chanie M. Comparative aspects of the clinical hematology of birds: a review. Br Poult Sci 2014;3:88-95. 
[15] Frankič T, Pajk T, Rezar V, Levart A, Salobir J. The role of dietary nucleotides in reduction of DNA damage induced by T-2 toxin and deoxynivalenol in chicken leukocytes. Food. Chem Toxicol 2006;44:1838-44. https://doi.org/10.1016/j.fct.2006.06.002

[16] Banchrof J, Steven A, Turner D. Theory and practice of histopathological techniques. Churchil Livingstone, New York, London, San Francisco, Tokyo; 1996.

[17] Desmet W, Van Hal B, Sas P, Vandepitte D. A computationally efficient prediction technique for the steady-state dynamic analysis of coupled vibro-acoustic systems. Adv Eng Softw 2002;33:527-40. https://doi.org/10.1016/S0965-9978(02)00062-5

[18] Pinto E, Vale-Silva L, Cavaleiro C, Salgueiro L. Antifungal activity of the clove essential oil from Syzygium aromaticum on Candida, Aspergillus and dermatophyte species. J Med Microbiol 2009;58:1454-62. https://doi.org/10.1099/jmm.0.010538-0

[19] Salehi B, Mishra AP, Shukla I, Sharifi-Rad M, Contreras MdM, SeguraCarretero A, et al. Thymol, thyme, and other plant sources: Health and potential uses. Phytother Res 2018;32:1688-706. https://doi.org/10.1002/ptr.6109

[20] Dibazar SP, Fateh S, Daneshmandi S. Clove (Syzygium aromaticum) ingredients affect lymphocyte subtypes expansion and cytokine profile responses: An in vitro evaluation. J Food Drug Anal 2014;22:448-54. https://doi.org/10.1016/j.jfda.2014.04.005

[21] Ahmad N, Alam M, Shehbaz A, Khan A, Mannan A, Hakim SR, et al. Antimicrobial activity of clove oil and its potential in the treatment of vaginal candidiasis. J Drug Target 2005;13:555-61. https://doi.org/10.1080/10611860500422958

[22] Joseph B, Sujatha S. Bioactive compounds and its autochthonous microbial activities of extract and clove oil (Syzygium aromaticum L.) on some food borne pathogens Asian J Biol Sci 2011;4:35-43. https://doi.org/10.3923/ajbs.2011.35.43

[23] El-Hack MA, Alagawany M. Performance, egg quality, blood profile, immune function, and antioxidant enzyme activities in laying hens fed diets with thyme powder. J anim Feed SCl 2015;24. https://doi.org/10.22358/jafs/65638/2015

[24] Gudev D, Popova-Ralcheva S, Moneva P, Ignatova M. Effect of the probiotic 'Lactina' on some biological parameters and nonspecific resistance in neonatal pigs. J Anim Sci Biotechnol 2008;24:87-96. https://doi.org/10.2298/BAH0802087G

[25] Toghyani M, Tohidi M, Gheisari AA, Tabeidian SA. Performance, immunity, serum biochemical and hematological parameters in broiler chicks fed dietary thyme as alternative for an antibiotic growth promoter. Afr J Mycol Biotechnol 2010;9:6819-25.

[26] Campbell T, Coles E. Avian clinical pathology. Vet Clin Pathol 1986;4:178 207.

[27] Abdelaziz M, El-Faham A, Ali NG. Using natural feed additives as alternative antimycotoxins in broiler diets. Egypt. Poult Sci.J 2015;35.

[28] Khoza S, Moyo I, Ncube D. Comparative hepatotoxicity of fluconazole, ketoconazole, itraconazole, terbinafine, and griseofulvin in rats. J Toxicol Clin Toxicol 2017;2017. https://doi.org/10.1155/2017/6746989

[29] Shalaby S. Magd El-Din M, A. Abo-donia S, Monir Mettwally, Attia A Z. Toxicological effects of essential oils from Eucalyptus Eucalyptus globules and clove Eugenia caryophyllus on Albino rats. Pol J Environ Study 2011;20:429-34.

[30] Liu Y, Jiang Y, Wu A, Chen S, Zhang Y, Liu M, et al. Prognostic significance of serum antioxidant parameters in immunocompetent patients with cryptococcal meningitis. Eur J Clin.Microbiol.Infect. Dis. 2012;31:235967. https://doi.org/10.1007/s10096-012-1577-y

[31] Dhama K, Chakraborty S, Verma AK, Tiwari R, Barathidasan R, Kumar A, et al. Fungal/mycotic diseases of poultry-diagnosis, treatment and control: a review. Pak J Biol Sci 2013;16:1626-40. https://doi.org/10.3923/pjbs.2013.1626.1640 